STUDI

FRANCESI

\section{Studi Francesi}

Rivista quadrimestrale fondata da Franco Simone

158 (LIII | II) | 2009

Varia

\title{
Jean-Luc Martinet, Montaigne et la dignité humaine. Contribution à une histoire du discours de la dignité humaine
}

\section{Luca Ruaro}

\section{(2) OpenEdition \\ Journals}

\section{Edizione digitale}

URL: http://journals.openedition.org/studifrancesi/7882

DOI: $10.4000 /$ studifrancesi.7882

ISSN: 2421-5856

\section{Editore}

Rosenberg \& Sellier

\section{Edizione cartacea}

Data di pubblicazione: 1 juillet 2009

Paginazione: 382-383

ISSN: 0039-2944

\section{Notizia bibliografica digitale}

Luca Ruaro, «Jean-Luc Martinet, Montaigne et la dignité humaine. Contribution à une histoire du discours de la dignité humaine», Studi Francesi [Online], 158 (LIII | II) | 2009, online dal 30 novembre 2015,

consultato il 08 janvier 2021. URL: http://journals.openedition.org/studifrancesi/7882 ; DOI: https:// doi.org/10.4000/studifrancesi.7882

Questo documento è stato generato automaticamente il 8 janvier 2021.

\section{cc) $($ ) $\ominus$}

Studi Francesi è distribuita con Licenza Creative Commons Attribuzione - Non commerciale - Non opere derivate 4.0 Internazionale. 


\title{
Jean-Luc Martinet, Montaigne et la dignité humaine. Contribution à une histoire du discours de la dignité humaine
}

\author{
Luca Ruaro
}

\section{NOTIZIA}

JEAN-LUC MARTINET, Montaigne et la dignité humaine. Contribution à une histoire du discours de la dignité humaine, Paris, Eurédit, 2007, pp. 316.

1 Tra i più recenti contributi ad una storia dell'idea occidentale di 'dignità umana' merita di essere segnalato questo saggio di Jean-Luc Martinet, che già in sede di presentazione mette opportunamente in rilievo come «la nostra modernità ricorra regolarmente a tale espressione, senza che sia sempre facile comprendere ciò che essa indica». A questo riguardo, in effetti, possiamo constatare come nel dibattito filosofico contemporaneo la nozione di dignità umana si sia venuta sostanzialmente ad articolare in due significati fondamentali, che sono tra loro connessi, anche se non risultano immediatamente e semplicisticamente sovrapponibili. Si possono cioè distinguere - essenzialmente - una 'teoria della dotazione', secondo cui la dignità umana è legata allo 'statuto ontologico' che sarebbe proprio di ogni essere umano; ed una 'teoria della prestazione', secondo la quale essa si ricollega piuttosto alla sua capacità di autonomia e autodeterminazione, configurandosi in definitiva come risultato dell'agire dell'uomo, ossia come una conquista della sua soggettività che si dà un'identità.

Il saggio di Martinet risulta di grande interesse anzitutto perché prende in considerazione proprio il momento decisivo nell'elaborazione del pensiero occidentale sulla dignità umana, e cioè l'età umanistico-rinascimentale: la cui svolta spirituale si può appunto ravvisare nello sforzo di portare al centro del dibattito culturale il 
discorso sull'uomo, soprattutto approfondendo e puntualizzando la nozione di dignitas hominis, in funzione della duplice eredità di pensiero pagana e cristiana. In particolare, nella celebre Oratio de hominis dignitate di Pico della Mirandola - vero e proprio manifesto della cultura rinascimentale - si manifesta «la novità di un atteggiamento mentale che non concepisce più quella dignità come insieme di doti ab aeterno attribuite all'uomo, connaturate col suo essere, ma la intende come libera conquista, come crescita interiore che ad ogni istante può venir compromessa, come qualità che ad ogni istante rischia di perdersi o sminuirsi o promette, invece, di esaltarsi e sublimarsi» (L. sozzi, Poliziano e la 'dignitas hominis' o l'elogio della dolcezza, "Rassegna Europea di Letteratura Italiana», 4/1994, pp. 143-144). D'altra parte, lo sconvolgimento dell'orizzonte culturale che caratterizza la seconda metà del Cinquecento avrebbe ben presto prefigurato, soprattutto attraverso gli Essais di Montaigne, una radicale 'ricostruzione' di ogni possibile discorso sulla dignità dell'uomo.

Questo dualismo di fondo trova una chiara ed efficace corrispondenza nelle due parti in cui si articola il saggio di Martinet. La prima parte (L'élaboration du discours de la 'dignitas hominis') mette anzitutto in rilievo come questa espressione nasca da una tradizione antica, riletta ed analizzata alla luce dell'influenza patristica ma anche ermetica, la cui importanza nel corso del Rinascimento è innegabile; sottolinea come in quest'epoca tale formula, irriducibile ad uno specifico campo intellettuale, si dissemini con facilità in tutti gli ambiti conoscitivi, assumendo il valore di un vero e proprio topos; e soprattutto evidenzia come questo discorso topico si fondi sull'affermazione incontestabile che fa dell'uomo l'immagine ed il possessore di un principio divino (che determina, peraltro, anche una strutturale opposizione tra la sua 'natura' e la sua 'condizione' reale). La seconda parte invece (Les "Essais": «Vivre à propos») è imperniata sulla figura di Montaigne e sui suoi Essais: che testimoniano mirabilmente, non solo nel contenuto ma nella loro stessa forma, la crisi che nella seconda metà del Cinquecento travolge le certezze e i valori consolidati, e più in generale la prospettiva filosofica tradizionale, capace di mantenere in costante rapporto tra loro la dimensione dell' 'Assoluto' (Dio) e quella del 'relativo' (l'uomo). In effetti Montaigne, con straordinaria sensibilità, avverte tutta la debolezza e la solitudine con cui l'uomo - del suo tempo, ma non solo - è costretto a confrontarsi nella complessa e spesso drammatica esperienza della sua vita: e in questa nuova prospettiva, evidentemente, egli non può più riproporre (magari con qualche variante) un discorso analogo a quello dei suoi predecessori, ma è costretto a ripensare la dignità nella sua integralità, giungendo così a definirla secondo criteri puramente umani. Questa ricostruzione radicale, dalle fondamenta, non sfocia dunque in un semplice approfondimento di quell'altra faccia della dignitas che - sempre secondo tradizione - era la miseria hominis, bensì nel rivoluzionario superamento della distinzione tra condizione umana e natura: nel senso che, per Montaigne, la dignità dell'uomo non si ricollega più alla sua somiglianza con Dio, ma sta piuttosto nella presa di coscienza e nell'accettazione della propria individualità, e nell'agire, conseguentemente, in modo "adeguato" al proprio essere limitato (p. 12: «La dignité humaine se métamorphose en convenable humain»). In modo del tutto corrispondente, d'altra parte, è la forma stessa degli Essais a creare «un diverso spazio enunciativo e discorsivo in seno al quale il nostro autore pensa l'uomo». Quanto poi all'interrogativo se si debba ravvisare in ciò un fallimento dell'Umanesimo, Martinet risponde in senso affermativo, almeno nella misura in cui gli Essais «abbozzano un nuovo discorso che consegna l'uomo alla sola libertà della condizione umana»: quella stessa libertà del resto - non esente da angoscia - che una parte della 
nostra modernità ha ripreso e fatto propria, e con la quale dunque ancor oggi siamo costretti a confrontarci. 